\title{
GERAÇÃO DE ESTILOS COGNITIVOS DE APRENDIZAGEM DE NEGOCIADORES EMPRESARIAIS PARA ADAPTAÇÃO DE ENSINO TUTORIALIZADO NA WEB ${ }^{1}$
}

\author{
Milton J.P. Madeira ${ }^{2}$ \\ Ricardo Wainer \\ Regina Verdin \\ João C. Alchieri \\ Eduardo K. Diehl \\ Universidade do Vale do Rio dos Sinos
}

\begin{abstract}
RESUMO: A presente pesquisa foi desenvolvida no cerne do projeto multidisciplinar e multinstitucional "Tapejara" - Sistemas Inteligentes de Ensino na Internet-. O artigo apresenta os Estilos Cognitivos de Aprendizagem gerados a partir dos resultados da aplicação do Teste Ross e da Bateria de Provas de Raciocínio BPR-5 numa amostra de funcionários da população-alvo do Curso de Negociação Empresarial a ser ministrado pela empresa parceira de telecomunicações. Os resultados da testagem cognitiva foram submetidos a análises fatoriais e, a seguir, a uma análise de cluster que, com seus respectivos dendogramas, possibilitaram o agrupamento da população em cinco grandes grupos a saber: Analógico-analítico, Concreto-genérico, Dedutivo-avaliativo, Relacional-sintético e Sintético-avaliativo. Os Estilos Cognitivos de Aprendizagem permitirão a Modelagem Cognitiva do Agente-Aprendiz que possibilitará um ensino tutorial adaptado às necessidades próprias de cada estilo cognitivo, e que assista eficazmente o aprendizado de forma assíncrona e individualizada.
\end{abstract}

Palavras-chave: Estilos Cognitivos, Tutores Inteligentes, Ensino a Distância, Negociação Empresarial.

\section{GENERATION AND CONCEPTUALIZATION OF COGNITIVE STYLES OF LEARNING OF ENTERPRISE NEGOTIATORS FOR ADAPTATION OF TUTORIAL TEACHING IN THE WEB}

\begin{abstract}
This article presents a research developed in the core of a multidisciplinary Project 'Tapejara' - Intelligent Systems of Instruction in the Internet -. The article presents, mainly, the Cognitive Styles of Learning generated from the results of Test Ross and BPR-5 Test applications in the employee's partner Company. The results from the cognitive assessment were submitted to a Cluster Analysis. The dendogramms allowed grouping the target population in five groups: Analogue-analytical, Concretegeneric, Evaluative-deductive, Relational-synthetic and Synthetic-evaluative. In the Project 'Tapejara', the Cognitive Styles of Learning will make possible to build the Student Model of the Pedagogical Agent, consequently will allow a tutorial adapted to the individual learning differences.
\end{abstract} Negotiators

Key-words: Cognitive Styles, Intelligent Tutoring System, Long-Distance Education Enterprise

Quando é solicitado a resolver tarefas cognitivas de aprendizagem no dia-a-dia, o ser humano tende a apresentar padrões de processamento da informação. Tais padrões mostram-se estáveis ao longo do

\footnotetext{
${ }^{1}$ Artigo recebido para publicação em fevereiro de 2002; aceito em abril de 2002

${ }^{2}$ Endreço para correspondência: Milton José Penchel Madeira, Curso de Psicoologia, Centro de Ciências da Saúde, Universidade do Vale do Rio dos Sinos/UNISINOS, Av. Unisinos, 950, C.P. 275, São Leopoldo, RS, Cep 93022-000, E-mail madeira@cirrus.unisinos.br
} 


\section{Milton J.P. Madeira}

tempo, e são denominados e ressaltados nas pesquisas em Ciências Cognitivas de Estilos Cognitivos de Aprendizagem. A geração e definição de tais estilos é parte constituinte do Projeto Multinstitucional Tapejara - Sistemas Inteligentes de Ensino na Internet -, o qual objetiva a construção e a avaliação de três tutores inteligentes de ensino via Web. A inteligência destes softwares consiste, fundamentalmente, no fato de possibilitar o reconhecimento do Estilo Cognitivo de Aprendizagem do usuário. Com isto, torna-se possível fornecer material didático adaptado às características de processamento de informação de cada estilo embutidos em Modelos de Aluno dos Tutores Inteligentes, buscando-se com isto, de acordo com os pressupostos da ergonomia cognitiva, gerar uma relação ensino-aprendizagem adaptada ao usuário e, portanto, mais eficaz (Ginsberg, 1993).

Estes estudos estão inseridos dentro da moderna área do conhecimento chamada Ciências Cognitivas, especificamente, dentro das pesquisas em Psicologia Cognitiva Experimental e em Inteligência Artificial.

Os Estilos Cognitivos de Aprendizagem são concebidos como padrões cognitivos estáveis que o indivíduo se utiliza no momento da sua aprendizagem, demonstrando serem viáveis para a elaboração de estratégias de ensino que levem em consideração características individuais do aprendiz e que gerem Modelos de Aluno-aprendiz nos Tutores Inteligentes. A deteç̧ão e/ou geração dos Estilos Cognitivos de Aprendizagem das populações-alvo no ensino via Web, mostra-se de grande significância, pois o desenvolvimento de tecnologias sofisticadas de ensino, como, por exemplo, o ensino assíncrono e a distância via web, deve levar sempre em consideração os pressupostos da ergonomia cognitiva. Estes dizem respeito à adaptação máxima das metodologias educacionais ao aprendiz, seja no que concerne às interfaces computacionais, seja no que se refere às características de aprendizagem do usuário.

É justamente nesta inovadora proposta de ensino-aprendizagem que o Projeto 'Tapejara' se concentra; buscando construir ferramentas computacionais que ensinem à distância e de forma inteligente ao reconhecer e se adaptar aos Estilos Cognitivos de Aprendizagem de cada usuário.

\section{Objetivos e Justificativa}

A pesquisa, que neste artigo tem seus resultados apresentados, teve como objetivos definir os procedimentos teórico-metodológicos de geração dos Estilos Cognitivos de Aprendizagem detectados na população-alvo de um daqueles três cursos previstos no Projeto "Tapejara", o de Negociação Empresarial da Empresa de Telecomunicações parceira no Projeto Tapejara. Isto se tornou possível, através da aplicação e avaliação dos resultados de Testes objetivos de investigação de processos cognitivos e de habilidades psicopedagógicas -Teste Ross (Ross \& Ross, 1997) e BPR-5 (Primi, 2000) - na população citada. Além de especificar os procedimentos de definição dos estilos, também foi definida e explicada a conceitualização dos mesmos. Buscou-se, igualmente, através das pesquisas realizadas, analisar a validade e fidedignidade do Teste Ross e do BPR -5 na avaliação de Estilos Cognitivos de Aprendizagem, em população gaúcha específica, gerando, secundariamente, a normatização destes instrumentos.

\section{Fundamentação Teórica}

\section{As Ciências Cognitivas}

As Ciências Cognitivas tratam do estudo multidisciplinar da natureza e do desenvolvimento da cognição em seres humanos, outros animais e em máquinas pensantes. Este estudo é implementado por parte de diversos campos do conhecimento que colaboram na busca de uma teoria unificada da cognição, orientados pelo paradigma da explicação e predição dos processos cognitivos. O fazer Ciência Cognitiva, teve assim, no estudo da inteligência e dos sistemas inteligentes, um de seus pontos mais importantes (Dunlop \& Fetzer, 1993; Madeira \& Filho 1995; Minsky, 1988; Simon \& Kaplan, 1993).

Conforme Madeira e Filho (1995):

"O objeto de estudo constituiu-se no estudo das cognições, como processos de inteligência (tanto sob o ponto de vista psicológico propriamente dito quanto no de simulação computacional), raciocínio, pensamento, resolução de problemas, criatividade e outros. Não mais meramente no sentido do estudo das estruturas destes processos, mas, fundamentalmente, ao nível dos 
processos que dizem respeito de maneira direta ou indireta acerca dos fenômenos de organização do conhecimento, seja através de arquiteturas naturais ou artificiais". (p. 233).

Diversos campos do conhecimento integram as Ciências Cognitivas dos quais podem ser citados a Psicologia Cognitiva Experimental, a Inteligência Artificial, a Psicopedagogia Cognitiva Experimental, a Filosofia (principalmente a epistemologia e a lógica), a Neurociência, a Linguística Cognitiva e a Robótica. Neste trabalho, constata-se a colaboração estreita de três dentre elas: Psicologia Cognitiva, Psicopedagogia Cognitiva e Inteligência Artificial.

Uma das investigações sempre presente no cerne dos trabalhos nestas disciplinas constituintes das Ciências Cognitivas, que também acaba por unificar este ramo da ciência, é o estudo da arquitetura dos sistemas inteligentes. A primeira das ciências que demarcou fortemente estes estudos foi a Inteligência Artificial, principalmente com os trabalhos de Simon (1981). Este autor precursava a possibilidade da simulação computacional de diversos sistemas, inclusive os ditos inteligentes, podendo assim vislumbrar a arquitetura destes sistemas. Esta idéia tem diversas argumentações que a embasam. Segundo Simon (1981), "devido ao seu caráter abstrato e à generalidade da sua manipulação de símbolos, o computador digital alargou grandemente a classe de sistemas cujo comportamento pode ser imitado" (p. 40).

Entre os métodos mais importantes criados ou adaptados pelas Ciências Cognitivas pode-se citar, a modelização axiomática, a simulação computacional, a análise de protocolos, o "thinking aloud" (pensar alto), a análise de conteúdo, a meta-análise, o mapeamento cognitivo e o estudo de lesados cerebrais.

Nas pesquisas do Projeto Tapejara, várias destas metodologias foram utilizadas para a obtenção dos objetivos almejados, em especial a modelização cognitiva, por sẹ o método que permitiu o objetivo maior deste estudo cognitivo, qual seja, a geração e definição de Estilos Cognitivos de Aprendizagem assim como a simulação computacional em Inteligência Artificial.
Esta fundamentação teórica se detém em descrever o estado da arte em duas disciplinas que sustentam toda a estrutura desta pesquisa e nas quais todo o trabalho está inserido, que são a Psicologia Cognitiva e a Inteligência Artificial

\section{Inteligência Artificial (IA)}

A denominação Inteligência Artificial (IA) originou-se em 1956, em uma reunião no College de Darmouth, na Inglaterra. Como foi visto no item anterior desta fundamentação, tal disciplina busca analisar os conhecimentos do homem, nos domínios da compreensão, da percepção e da resolução de problemas, a fim de os reproduzir, com a ajuda das máquinas em simulações realizadas por programas (Ginsberg, 1993; Minsky, 1988, apud Oliveira, 1996; Rich \& Knight, 1994; Pitrat, 1985, apud Silveira, 1992).

Na Psicologia Cognitiva, a IA tem colaborado muito no sentido de exigir constantes desenvolvimentos e reformulações em posições teóricas sobre vários aspectos do conhecimento sobre os processos psíquicos superiores, pois que no momento da implementação computacional em que se traduzem os conhecimentos de linguagem natural para linguagem lógica (típica e apropriada à IA), qualquer inconsistência dos dados emerge, fazendo com que o pesquisador em Psicologia Cognitiva tenha de rever seus resultados.

Além disso, muitos conceitos e processos estudados em seres humanos pela Psicologia Cognitiva são estudados pela Inteligência Artificial em computadores. Estes processos que se mostram na interseção das duas disciplinas são, por exemplo, o processo de aquisição de conhecimento (semântica, conceitos), os processos de inferência, o processo de resolução de problemas, as estratégias de resolução de problemas, os tipos de raciocínio (indutivo e dedutivo), os processos metacognitivos (Genesereth \& Nilson, 1988; Medin \& Ross, 1992). Todos estes estudos, vale salientar, são abordados na IA por autores que, muitas vezes, têm concepções distintas sobre a natureza da informação. Por exemplo, há divisões entre os pesquisadores, categorizados em logicistas, conexionistas, moboticistas (Kirsh, 1991). 
Portanto, a IA constitui-se em ferramenta importante para o desenvolvimento do conhecimento nas mais diversas áreas da Psicologia Cognitiva, seja por sua contribuição em termos das simulações computacionais de diversos processamentos cognitivos, em especial os de aprendizagem, seja pela possibilidade de construção de tutores inteligentes.

\section{Psicologia Cognitiva}

Os estudos que possibilitam geração de Estilos Cognitivos de Aprendizagem foram realizados no âmbito da Psicologia Cognitiva sob o enfoque do processamento da informação (Sternberg, 2000; Stillings et. al., 1991). Esta, surgiu em meados da década de 60', sendo considerada uma Psicologia Pós-Behaviorista, pelo seu caráter empírico, teórico, metodológico e científico, mas contrapondo-se ao Behaviorismo no sentido de não limitar seus estudos aos modelos estímulo (S)- resposta (R) ou $S$ - organismo (O) - R. Ela busca, pela observação sistemática do comportamento, pela engenhosidade experimental e até mesmo pela simulação computacional advinda da sua ciência irmã, a Inteligência Artificial, estudar a vida mental e predizer como se dão os processos mentais e qual a arquitetura destes. A Psicologia Cognitiva tem assim o propósito de desvendar o que ocorre na "caixa-preta" do psiquismo humano (Madeira, 1989).

Vários autores comungam destas idéias básicas. Para Costermans (1981), a Psicologia Cognitiva se refere ao estudo experimental dos processos de tratamento da informação, através dos quais o sistema nervoso assegura a gestão do comportamento.

Ressalta-se que o termo "informação", utilizado pela Psicologia Cognitiva sob a abordagem do Processamento da Informação, e de crucial importância para a gênese e desenvolvimento tanto da Psicologia Cognitiva quanto de todas as Ciências Cognitivas, foi formalizado a primeira vez por Shannon (1948), que se preocupava com o desenvolvimento de teorias matemáticas sobre a comunicação e como evitar os "ruídos" nos canais da comunicação (Abrantes, 1993; Medin \& Ross, 1992; Pozo, 1998). Informação, então, foi considerada, segundo Shannon (1948), como sendo a redução da incerteza.
Norbert Wiener ressalta a importância deste novo conceito, referindo que informação é informação, não matéria ou energia e complementa afirmando que nenhum materialismo que não admita isto pode sobreviver nos dias atuais (Wiener, 1961, apud Gardner, 1995). É justamente esta concepção de informação de Shannon (1948) que o presente trabalho assumiu.

Atualmente, os estudos em Psicologia Cognitiva têm voltado sua atenção para os padrões de processamento da informação do ser humano, quando é solicitado a resolver tarefas cognitivas as quais é submetido no cotidiano. Tais padrões, que em última instância refletem a maneira das pessoas gerenciarem-se nas mais diversas situações, são chamados de Estilos Cognitivos de personalidade.

\section{Estilos Cognitivos de Personalidade}

O conceito de Personalidade é um dos mais populares na Psicologia, mas que apresenta muitas dificuldades de conceituação. Apesar disto, assume-se, de modo geral, que personalidade é o conjunto de características mais típicas do indivíduo em sua interação com o ambiente; incluindo características comportamentais e cognitivas. Se pensarmos, portanto, através de uma abordagem cognitivista, personalidade é o modo característico como o sujeito processa as informações, determinando seu estilo de gerenciar-se nas mais diversas situações.

Pode-se perceber que o conceito de personalidade tem intrinsecamente a característica de estabilidade, ou seja, a personalidade não é algo mutável a cada momento. Possui consistência e durabilidade (Bagley \& Malick, 1998; Clarke, 1998).

Uma das inovações teóricas mais importantes ocorrida neste campo foi a conceitualização de Estilos Cognitivos de personalidade (Riding \& Rayner, 2000; Chanlin, 1998). A Psicologia Cognitiva buscou identificar e definir os padrões característicos de interações entre os processos cognitivos superiores (como raciocínio, resolução de problemas, memória, formação de conceitos mentais etc) que geravam um modo individual e estável de processamento mental em que uma parcela significativa de pessoas acabavam utilizando sis- 
tematicamente em suas vidas. Os diferentes padrões característicos e estáveis de processar informação constituem os Estilos Cognitivos de personalidade (Hunt, 1999).

Estilos Cognitivos caracterizam heurísticas de alto nível que organizam e gerenciam o comportamento do indivíduo, durante a sua aprendizagem. O estilo cognitivo é, portanto, uma das características mais constantes do indivíduo e se apresenta em padrões estáveis de processamento da informação. Segundo Fierro (1990, apud Salvador et al., 2000), Estilos Cognitivos podem ser definidos como padrões diferenciais e individuais de reação diante da estimulação recebida, de processamento cognitivo da informação e, decididamente, de enfrentamento cognitivo da realidade. A estabilidade de um estilo cognitivo se manifesta nas hierarquias de utilização de processos de tratamento da informação e das estratégias próprias que o aprendiz utiliza quando adquire uma nova informação.

Quando define os Estilos Cognitivos Messick (1976) fala em modos habituais dos seres humanos processarem a informação, representando um modo característico do aprendiz de perceber, pensar, e resolver problemas. Os Estilos Cognitivos também podem ser definidos, conforme Sternberg (1992), como a forma pela qual a pessoa codifica e decodifica a informação por meio de operações mentais, tais como codificação seletiva, combinação seletiva e comparação seletiva.

Um dos objetivos da determinação dos Estilos Cognitivos está justamente em se poder ajustar o material didático e os ambientes de aprendizagem às diferenças individuais, a fim de gerar modos mais eficazes e adaptados para as características de aprendizagem dos sujeitos.

Sendo uma das áreas mais pesquisadas pelas Ciências Cognitivas, a Aprendizagem também acabou aproveitando este constructo psicológico em suas investigações. Inicialmente, buscando verificar de que modo a aprendizagem seria facilitada se fossem considerados os estilos de personalidade do aprendiz.

Com os avanços obtidos pelas pesquisas, verificou-se que se poderia falar em Estilos Cognitivos de Aprendizagem, ou seja, os modos diferenciais que os indivíduos desenvolvem para processar informação quando submetidos a tarefas de aprendizagem com que se defrontam no seu cotidiano (Riding \& Rayner, 2000).

\section{Estilos Cognitivos de Aprendizagem}

Estilos Cognitivos de Personalidade são padrões diferenciais de reações, relativamente estáveis, diante de uma estimulação recebida, via processamento cognitivo da informação e do enfrentamento cognitivo da realidade.

Já os Estilos Cognitivos de Aprendizagem dizem respeito a uma das dimensões dos Estilos Cognitivos de Personalidade. Enquanto que, ao se falar de Estilos Cognitivos engloba-se características emocionais e afetivas, típicas da Personalidade do indivíduo; ao se falar de Estilo Cognitivo de Aprendizagem estamos nos restringindo ao modo característico do sujeito de aprender novos conceitos ou mesmo de gerar elaborações a partir de um conhecimento prévio (Becker, 1991; Mckay, 1999; Schmeck, 1988).

Torna-se importante salientar que a teorização sobre os Estilos Cognitivos de Aprendizagem está quase que diretamente associada aos estudos de ensino a distância e de tutores inteligentes (Nassar, 1999). Isto ocorre porque se podem gerar modelos de aluno nos softwares de Inteligência Artificial pela determinação de tais Estilos Cognitivos. Estes softwares fornecem o material instrucional adaptado às diferenças individuais, aumentando, portanto, a eficiência do processo de ensino.

O presente artigo apresenta a geração e conceitualização de cinco Estilos Cognitivos de Aprendizagem advindos da investigação de uma população-alvo específica, a qual será beneficiada com um ensino proporcionado por um tutor inteligente via Web. Acredita-se, pois, que para além dos avanços obtidos dentro dos objetivos gerais do Projeto 'Tapejara', tenha-se avançado também no sentido de contribuir com uma metodologia para a geração de Estilos Cognitivos de Aprendizagem. Esta contribuição se dá também no que se relaciona aos processos cognitivos e habilidades psicopedagógicas levadas em consideração para o agrupamento dos estilos.

Pode-se afirmar, deste modo, que a Psicologia Cognitiva consegue elaborar um estudo comprometido com o método científico e em pro- 


\section{Milton J.P. Madeira}

fundidade da mente humana. Esta ciência utilizase de modelos lógico-matemáticos que permitem a investigação de fatores que, anteriormente, eram dificilmente pesquisados pelos métodos meramente observacionais disponíveis. Possibilita-se assim que se pesquise temas como os Estilos Cognitivos de Aprendizagem e a Negociação Empresarial, objetos deste artigo.

\section{As Pesquisas sobre Negociação Empresarial}

Os estudos sobre Negociação abrangem três tradições teóricas principais. A primeira tradição consiste na elaboração de 'conselhos' para o negociador, escritos no séc. XVII por Callières (Carnevale e Pruitt, 1992). Há escritos mais atuais dentro desta linha direcionados para a negociação industrial e internacional, elaborados por Nicolson, e Peters (Carnevale e Pruitt, 1992). Nas últimas décadas, as produções teóricas se direcionaram para a investigação sob um ângulo econômico com cargos específicos como gerentes (Lax \& Sebenius, 1986) ou para negociadores em geral (Carnevale \& Pruitt, 1992).

A segunda tradição envolve a construção de modelos racionais e matemáticos na Negociação e Mediação, elaborados por economistas e teóricos do jogo Harsanyi, Nash, Zeuthen (Carnevale \& Pruitt, 1992). Estes modelos, considerados normativos, especificam normas e regras com os quais os negociadores podem atuar ou especificam como deve ser a dinâmica do comportamento do negociador.

A terceira tradição, também denominada tradição comportamental, tem suas raízes teóricas inicialmente nos modelos matemáticos, e depois se direcionou à análise de comportamentos em negociações sindicais (Thompson, 1998).

Nas duas últimas décadas os estudos na área comportamental em negociação focalizaram os mecanismos sociais-cognitivos, que englobam pesquisas em Cognição Social de Sherman et al (1989), o processamento de informação na Psicologia Cognitiva de Anderson (1985) e Teoria da Decisão de Dawes (1988), orientada para negociação.

Neste panorama geral, as pesquisas em $\mathrm{Ne}$ gociação colocam em foco o negociador e seguem a definição de Negociação.

\section{Definições de Negociação}

Para Thompson (1998) Negociação é um processo de tomada de decisão interdependente em que fazem parte os seguintes componentes: julgamento individual, a interdependência e a cooperação. É principalmente uma tarefa em que se processam informações combinando a aquisição dessas informações, análises e tomada de decisão.

Bazerman e Neale (1992) também definem a negociação como um processo de Tomada de Decisão entre partes que são interdependentes e que não compartilham os mesmos interesses. Por meio da negociação, as partes envolvidas decidem as melhores opções ou curso de ação a serem seguidos. A negociação nesse sentido é um processo de tomada de decisão que se caracteriza pela interdependência entre as partes que negociam e que realizam acordos. Em ambas definições, portanto, considera-se a negociação como um processo de tomada de decisão.

\section{Método}

\section{Amostra}

A amostra avaliada nesta pesquisa foi composta por 534 funcionários, de uma empresa dè telecomunicações do Rio Grande do Sul, que é o público-alvo do curso de Negociação Empresarial. Desta população foram selecionados 81 sujeitos para serem testados, por um processo de amostragem de escolha aleatória proporcional à distribuição dos funcionários nos diferentes setores da empresa.

\section{Procedimento}

Os dados foram coletados num período de um mês, em sessões coletivas de aplicação com 25 sujeitos em cada encontro, os quais tinham duração aproximada de uma hora e meia. $O$ setting de aplicação constituiu-se numa sala na própria empresa especialmente destinada às testagens, com iluminação artificial, ventilação e excelente controle de variáveis intervenientes externas (como barulho e interrupções, por exemplo), conforme preconiza Pasquali (1997). Os aplicadores foram psicólogos devidamente treinados no procedimento de aplicação dos instrumentos. Obtevese assim, uma elevada padronização das testagens, 
sendo que o psicólogo que ficava responsável por cada um dos encontros de aplicação do Teste Ross e do teste BPR - 5, executava o rapport inicial, bem como a leitura das instruções do manual do teste, segundo padronização proposta pelos autores de ambos os testes (Ross \& Ross, 1997; Primi, 2000).

\section{Instrumentos}

Os instrumentos utilizados para a avaliação psicológica foram o Teste Ross de Processos Cognitivos (Ross \& Ross, 1997), o qual foi adaptado e validado para a população em questão, bem como três subtestes da Bateria de Provas de Raciocínio (BPR-5) (Primi, 2000). Este último foi utilizado com o propósito de checagem mais fina das habilidades verificadas nos resultados do Teste Ross, tendo em vista que em trabalho anterior desta mesma equipe, 31 sujeitos não foram classificados em nenhum dos quatro estilos gerados e, assim sendo, a utilização do BPR-5 justificou-se como uma forma de aumentar a capacidade de discriminação dos Estilos Cognitivos de Aprendizagem.

O Teste Ross é apoiado na Taxonomia de Bloom (1972), em que os processos cognitivos possíveis de serem avaliados são: Raciocínio Analógico, Premissas Ausentes e Análise de Informação Relevante e Irrelevante (compondo a habilidade psicopedagógica de Análise), Relações Abstratas, Síntese Seqüencial e Análise de Atributos (compondo a habilidade de Síntese), os processos de Raciocínio Dedutivo e Estratégias de Questionamento (compondo a habilidade de Avaliação). Tais processos são subjacentes às habilidades consideradas psicopedagógicas também avaliadas pelo Teste Ross (Análise, Síntese e Avaliação).

As três Habilidades Psicopedagógicas que são avaliadas, portanto, no Teste Ross, podem assim ser definidas:

Análise: os processos envolvidos na análise sugerem uma ampliação da compreensão da finalidade do material, a evocação e a generalização. A hierarquia relativa de idéias é tornada clara e/ou as relações entre idéias expressas são tornadas explícitas.

Sintese: observa-se uma união de elementos e partes, de modo a formar um todo, numa estrutura não percebida claramente antes.
Avaliação: processo de julgamento acerca do valor de idéias, trabalhos, soluções, métodos, etc, realizados com um determinado propósito. $\mathrm{Na}$ taxionomia, no domínio cognitivo, ela é considerada um estágio final do complexo processo que envolve certa combinação dos elementos anteriores, embora não seja o último estágio do pensamento ou solução de problemas.

\section{Definições dos Processos Cognitivos Avaliados no Teste Ross}

Processos cognitivos são, basicamente, atividades cognitivas representacionais que acontecem na memória, envolvendo a codificação ou os processos de pensamento. Quando esses processos de pensamento são estudados pela Psicologia Experimental Cognitiva, eles podem ser descritos em termos de habilidade que os indivíduos consistentemente utilizam ao realizar certos tipos de tarefas (tais como testes psicológicos) e, então, são referidos como 'habilidade cognitivas' (Schmeck, 1988). Para avaliar as três habilidades psicopedagógicas (análise, síntese e avaliação), o Teste Ross investiga os processos cognitivos subjacentes a cada uma delas nas oito seções do teste. Uma descrição mais pormenorizada de cada um destes processos pode ser visto em (Madeira et. al., no prelo).

\section{Quanto a Abrangência do Teste Ross}

O Teste Ross busca compreender o como os sujeitos processam a informação, de uma maneira mais ampla, pois os itens do teste requerem do sujeito a realização de uma variedade de atividades para que, através dos dados avaliados, seja possível detectar um espelhamento de suas habilidades cognitivas (Ross \& Ross, 1997). Deve-se notar, entretanto, que o Teste Ross foi cuidadosamente elaborado para concentrar sua principal ênfase nas habilidades dos indivíduos para tratar com abstrações a partir de uma base verbal. Isto porque a habilidade para elaborar conceitos a partir de abstrações com elementos verbais não perde a afinidade com a realidade componente central dos processos cognitivos superiores.

\section{Quanto à Validade e à Fidedignidade do Teste Ross}


Qualquer instrumento de pesquisa precisa ser avaliado a fim de estabelecer se ele é capaz de discriminar, medir e investigar de forma consistente o que se propõe (Pasquali, 1996). Para isso existe o teste estatístico alpha de Cronbach que mede a consistência interna dos dados e estabelece em que medida ele é discriminativo ou não. $O$ alpha de Cronbach é uma medida cujos valores variam entre zero e um, sendo que zero é a consistência nula, e um, a consistência absoluta. Nas ciências sociais admite-se um alpha de 0,75 é bastante satisfatório e adequado para a aceitação de um instrumento como viável para pesquisas.

O Teste Ross foi testado em três níveis:

1. Ao ser testado pelos 105 itens (105 questões que compõem o teste) obtivemos um alpha de Cronbach de 0,91.

2. Ao ser testado pelos oito processos cognitivos obtivemos um alpha de Cronbach de 0,79 .

3. Ao se testado pelos três habilidades psicopedagógicas obtivemos um alpha de Cronbach de 0,80 .

Conclui-se, portanto, que o Teste Ross, para a amostra trabalhada, foi altamente discriminativo, consistente e fidedigno.

\section{Quanto à Normatização do Teste Ross para a População da CRT}

A fim de obter resultados que fossem realmente válidos para a população alvo, foi desenvolvido todo um trabalho de adaptação do Teste Ross às especificidade desta população.

A distribuição dos indivíduos em faixas de desempenho, a saber: Inferior (I), Inferior a Termo Médio (ITM), Termo Médio (TM), Superior a Termo Médio (STM), Superior (S), levou em conta o desempenho diferenciado da amostra em diferentes testes dentre os oito testes utilizados, e não o número de questões em cada seção. Deu-se, portanto, a estas faixas de desempenho, uma validade indiscutível.

Os mesmos processos de análise estatística e os mesmos cuidados metodológicos foram tomados ao estabelecermos faixas de desempenho nas três habilidades psicopedagógicas.

\section{Bateria de Provas de Raciocínio 5 (BPR-5)}

A Bateria de Testes de Raciocínio (BPR 5) é um instrumento para avaliação simultânea do raciocínio geral e de aptidões específicas. Esta bateria pretende avaliar a capacidade de raciocínio dos indivíduos, fortemente associada a um fator geral $(\mathrm{g})$, comum a toda atividade mental e envolvendo a conjugação de três operações mentais: a apreensão da informação, a edução de relações e a edução de correlatos (generalização), recorrendo a provas de conteúdos diversos. Nesse caso, e já em uma linha de aptidóes diferenciadas, as provas avaliam o raciocínio por itens de conteúdo numérico $(R N)$, verbal ( $R V)$, figurativo abstrato (RA), visual espacial (RE) e mecânico (RM).

O BPR - 5 é composto, portanto, por cinco provas, das quais três foram utilizadas para atingir o objetivo deste trabalho, uma vez que estas três provas são as que realmente auxiliam na determinação dos Estilos Cognitivos de Aprendizagem da população. Além disso, havia a preocupação de se realizar uma avaliação criteriosa da amostra, mas preservando os sujeitos de uma bateria de testes muito extensa que poderia se tornar extenuante.

\section{Justificativas da Aplicação das três provas da Bateria BPR-5}

Decidiu-se por aplicar, nesta amostra do curso de negociação, dois testes: o Teste Ross e a Bateria de Provas de Raciocínio (BRP-5). A justificativa para isto se sustenta no fato da amostra do Curso de Telecomunicações e Tecnologia SDH, avaliada em pesquisa anterior do grupo (Madeira et al., no prelo), não ter sido totalmente explicada pelo Teste Ross utilizado, anteriormente, como único instrumento. Naquela ocasião, cerca de $16 \%$ da amostra (31 sujeitos) mostrou não se enquadrar em nenhum Estilo Cognitivo gerado a partir da mensuração pelo Teste Ross, ou seja, este percentual da amostra não se caracterizou dentre os Estilos Cognitivos elaborados a partir dos dados do Teste Ross.

As hipóteses levantadas para explicar estes dados se baseiam no fato do clima organizacional, no momento das aplicações, ter exercido algum efeito nos sujeitos, uma vez que naquele momento havia ùm processo interno de mudanças administrativas. Estas mudanças geraram um certo clima de apreensão e insegurança nos sujeitos examinados, fazendo com que o nível de motivação e seriedade destes sujeitos na aplicação nạo tenha sido o ideal, conforme os dados de uma investigação 
exploratória a respeito das motivações dos sujeitos daquela amostra (Madeira, Carneiro et al., 2002).

Através da aplicação do BPR-5, gerou-se a possibilidade estatística de verificar a fidedignidade da aplicação, excluindo os sujeitos que não realizaram o Teste com seriedade, $e$ assegurando a confiabilidade da mesma. Por esta razão optou-se pela utilização do BPR-5 que possibilitou ainda a utilização de mais dados para fazermos correlações com o Teste Ross. Além disso, a populaçãoalvo do Curso de Negociação é mais heterogênea em relação à população dos dois primeiros cursos que era formada, basicamente, por engenheiros e técnicos em eletrônica. No Curso de Negociação Empresarial (conteúdo instrucional de um dos tutores inteligentes previstos no Projeto Tapejara), profissionais das mais diversas áreas farão parte da amostra, gerando, hipoteticamente, Estilos Cognitivos de Aprendizagem ainda mais diversos entre si.

A utilização conjunta do BPR-5 com o Teste Ross acabou, efetivamente, por garantir a fidedignidade e a validade da avaliação.

Assim sendo, as três provas utilizadas foram:

Prova de Raciocínio Abstrato (Prova RA): esta prova avalia a capacidade de raciocinar em situações novas, criar conceitos e compreender implicações. Composta por 25 itens de conteúdo abstrato, envolvendo analogias com figuras geométricas de acordo com a relação $\mathrm{A}: \mathrm{B} / / \mathrm{C}:(\mathrm{A}, \mathrm{B}, \mathrm{C}$, $D, E)$. Os sujeitos têm, então, a tarefa de descobrir a relação existente entre os dois primeiros termos e aplicá-la ao terceiro, para se identificar a quarta figura entre as cinco alternativas de resposta. $\mathrm{O}$ tempo limite é de 12 minutos.

Prova de Raciocínio Verbal (Prova $R$ V): avalia a extensão e a profundidade do conhecimento verbal vocabular e a capacidade de utilizar conceitos previamente aprendidos. É composta de 25 itens, envolvendo analogias entre palavras. A relação análoga entre um par de palavras deverá ser descoberta e aplicada de forma que identifique a quarta palavra, entre as cinco alternativas de resposta, mantendo a mesma relação com uma terceira apresentada. Nesta prova o tempo limite é de 10 minutos.
Prova de Raciocínio Espacial (Prova RE): nesta prova se avalia a capacidade de processamento visual, definida como a habilidade de representar e manipular imagens mentais. vinte itens compõem esta prova, onde existem séries de cubos tridimensionais em movimento. Descobrindo-se o movimento, através da análise das diferentes faces dos cubos, deve-se escolher, entre as alternativas de resposta, a representação do cubo que se seguiria se o movimento descoberto fosse aplicado ao último cubo da série. O tempo limite é de $18 \mathrm{mi}-$ nutos.

\section{Quanto à Normatização do BPR-5 para a Popu- lação da CRT}

Assim como foi feito com relação ao Teste Ross, os dados coletados pelo BPR-5 também foram tratados estatisticamente com vistas à obtenção de resultados realmente válidos para a população alvo, adaptando este teste, assim, às especificidade desta população.

Como no Teste Ross, os escores dos sujeitos foram divididos por faixas de desempenho a saber: Inferior (I), Inferior a Termo Médio (ITM), Termo Médio (TM), Superior a Termo Médio (STM), Superior (S). Esta divisão levou em conta o desempenho diferenciado da amostra em diferentes sub-testes e não o número de questões em cada seção, validando estas faixas de desempenho. Assim sendo, por exemplo, considerando-se a prova de Raciocínio Verbal que apresenta uma variação de 10 a 24 pontos, um participante que atingiu escore entre 15,7 e 18,4 se enquadrou na faixa Termo Médio (TM).

\section{Quanto às Análises de Correlação Realizadas entre os Testes Utilizados}

Os resultados das correlações entre os Teste Ross e as três provas da Bateria BPR-5, apresentados no quadro 5 , denotam correlações significativas (até muito significativas) entre praticamente todos os Subtestes do Teste Ross e as três provas do BPR-5 utilizadas. Isto indica que houve boa qualidade na testagem psicológica realizada, o que, de certa maneira também pode ser percebido pelo enquadramento de $95 \%$ da amostra dentro dos cinco Estilos Cognitivos de Aprendizagem retirados das Análises de Clusters, os quais podem ser visualizados no quadro 01 . 
Quadro 01: Correlação entre Resultados do Teste Ross e Subtestes da Bateria de Provas de Raciocínio BPR-5

\begin{tabular}{|c|c|c|c|c|c|c|c|c|c|}
\hline & \multicolumn{3}{|c|}{ Raciocínio Verbal } & \multicolumn{3}{|c|}{ Raciocínio Abstrato } & \multicolumn{3}{|c|}{ Raciocínio Espacial } \\
\hline & Correlação & $\begin{array}{c}\mathbf{P} \\
\text { (2-tailed) }\end{array}$ & $\begin{array}{c}\text { Sig. } \\
\text { (2-tailed) }\end{array}$ & Correlação & $\begin{array}{c}\mathbf{P} \\
\text { (2-tailed) }\end{array}$ & $\begin{array}{c}\text { Sig. } \\
\text { (2-tailed) }\end{array}$ & Correlação & $\begin{array}{c}\mathbf{P} \\
\text { (2-tailed) }\end{array}$ & $\begin{array}{c}\text { Sig. } \\
\text { (2-tailed) }\end{array}$ \\
\hline Raciocínio Dedutivo &, 468 &, 000 & MS & ,346 &, 002 & $\mathrm{MS}$ & 455 &, 000 & $\mathrm{MS}$ \\
\hline Premissas Ausentes & ,358 &, 001 & MS & 309 &, 005 & MS &, 478 &, 000 & MS \\
\hline Relações Abstratas &, 230 &, 041 & $S$ &, 111 &, 325 & NS & ,294 &, 008 & NS \\
\hline Síntese &, 163 &, 149 & NS &,- 013 & ,908 & NS & ,229 &, 041 & $S$ \\
\hline $\begin{array}{l}\text { Estratégia de Questi- } \\
\text { onamento }\end{array}$ &, 371 &, 001 & MS &, 198 & ,079 & NS & ,475 &, 000 & MS \\
\hline $\begin{array}{l}\text { Análise da Informa- } \\
\text { ção Relevante e Irre- } \\
\text { levante }\end{array}$ &, 504 &, 000 & MS & 280 &, 012 & $S$ &, 570 &, 000 & MS \\
\hline Análise & 199, & ,077 & NS & ,254 & ,023, & S &, 354 & ,001 & MS \\
\hline Analogias &, 301 &, 007 & MS &, 355 &, 001 & MS & ,518 &, 000 & MS \\
\hline Raciocínio Dedutivo &, 454 &, 000 & MS &, 352 &, 001 & MS & ,457 &, 000 & MS \\
\hline
\end{tabular}

Nota. "MS" Correlação é muito significante ao nível de .01 (2-tailed). "S" Correlação é significante ao nível de .05 (2-tailed).

"NS" Correlação não significante.

\section{Quanto à Validade e à Precisão do Teste BPR-5}

$A$ análise dos itens, do índice de dificuldade e do poder discriminativo do BPR-5, indica que as provas cumprem os requisitos necessários à composição de escalas precisas. Os itens apresentam boa variância e se correlacionam com o escore total. Isto indica que estes itens estão consistentes dentro das diferentes provas e contribuem para a variância do escore, produzindo coeficientes de precisão adequados.

O Teste BPR-5 foi testado em dois níveis:

1. Ao ser testado pelos seus 70 itens referentes às três provas ( 25 itens da prova Raciocínio Abstrato, 25 itens da prova Raciocínio Verbal e 20 itens da prova Raciocínio Espacial) obtivemos um alpha de Chronbach de 0,89.

2. Ao ser testado pelas três provas, obtivemos um alpha de Cronbach de 0,78.

Concluiu-se, portanto, que as provas utilizadas do Teste BPR-5, para a amostra trabalhada, foram altamente discriminativas, consistentes $\mathrm{e}$ fidedignas.

\section{Resultados}

As análises dos dados foram realizadas em três momentos. No primeiro momento, foi verificada a confiabilidade dos instrumentos pela sua consistência interna. Num segundo momento, realizou-se uma análise fatorial, na qual se levantou os fatores explicativos da variação dos resul- tados. Finalmente foi ultimada a especificação por fator e o agrupamento dos dados por clusters.

\section{Quanto às Análises Descritivas dos Dados}

No quadro 2, pode-se ter uma visão geral do desempenho da amostra em cada um dos 8oito processos cognitivos avaliados pelo Teste Ross e nas três habilidades mensuradas no BPR-5.

Quadro 2: Médias Gerais dos Oito Processos Cognitivos / Resultados Amostra Negociação Médias Gerais nos Oito Processos/ Ordem Original (no Teste de Ross) e Médias Gerais nas Três Habilidades do Teste BPR-5

\begin{tabular}{|c|c|c|}
\hline Teste Ross (Subtestes) & Média & $\begin{array}{c}\text { Desvio Pa- } \\
\text { drão }\end{array}$ \\
\hline 1.Analogias & 10,60 & 2,11 \\
\hline 2.Raciocínio Dedutivo & 14,09 & 3,26 \\
\hline 3.Premissas Ausentes & 5,51 & 1,52 \\
\hline 4.Relações Abstratas & 12,01 & 2,68 \\
\hline 5.Síntese Seqüencial & 3,46 & 2,61 \\
\hline 6.Estratégias de Questionamento & 9,34 & 2,65 \\
\hline $\begin{array}{l}\text { 7.Análise de Informações } \\
\text { Relevantes e Irrelevantes }\end{array}$ & 8,72 & 2,28 \\
\hline 8.Análise Atributos & 9,85 & 3,17 \\
\hline BPR-5 (Subtestes) & Média & $\begin{array}{l}\text { Desvio Pa- } \\
\text { drão }\end{array}$ \\
\hline 1. Raciocínio Verbal & 19,33 & 2,94 \\
\hline 2.Raciocínio Abstrato & 15,28 & 3,56 \\
\hline 3. Raciocínio Espacial & 11,28 & 4,68 \\
\hline
\end{tabular}




\section{Quanto às Análises Fatoriais Realizadas}

Realizou-se análises fatoriais, tanto dos dados advindos do Teste Ross quanto dos advindos do BPR-5, a fim de verificar o quanto cada um dos fatores - processos cognitivos e habilidades psicopedagógicas (para o Teste Ross) e as três provas utilizados do BPR-5 explicavam a variância total dos dados. Tal procedimento foi de crucial importância para a geração da nomenclatura dos Estilos Cognitivos de Aprendizagem.

A variância total explicada pelos oito processos cognitivos do Teste Ross foi de 44,26\%, sendo o Raciocínio Dedutivo o processo com maior percentual $(8,30 \%)$.

Em relação às três habilidades psicopedagógicas do Teste Ross, a variância total explicada foi de $71,72 \%$, sendo que praticamente todas as três habilidades tiveram o mesmo peso (Análise 25,13\%; Síntese - 20,76\% e Avaliação - 25, $83 \%)$.

Quanto à variância explicada pelas três provas utilizadas do BPR-5, teve-se um percentual de 71,39\%, assim divididos: Raciocínio Verbal (22,70\%); Raciocínio Abstrato (24\%) e Raciocínio Espacial $(24,69 \%)$.

\section{Quanto aos Agrupamentos por Semelhanças (determinação dos Estilos Cognitivos de Apren- dizagem)}

A análise de Cluster permite agrupar os indivíduos pela similaridade no desempenho em cada item do teste, processo ou habilidade avaliada. É do interjogo entre a análise fatorial e estes agrupamentos por similaridade que emergem os Estilos Cognitivos de Aprendizagem.

A variância dos resultados foi explicada pe- los oito fatores que correspondem aos oito processos cognitivos. Os sujeitos foram agrupados por níveis de semelhança na análise dos clusters num intervalo de $(0-5)$ dentro do Dendograma, sendo que quanto mais próximo de (0), mais importante.Foram determinados cinco agrupamentos bastante distintos.

No Quadro 03, temos os resultados desta análise, sendo que o quadro está organizado da seguinte maneira:

$I^{a}$ Coluna: os cinco Estilos Cognitivos de Aprendizagem gerados.

$2^{a}$ Coluna: o número de participantes da amostra que se agruparam dentro de cada Estilo.

$3^{a}$ e $4^{a}$ Colunas: o percentual da amostra e o percentual cumulativo nos estilos, respectivamente.

$5^{a}, \sigma^{a}$ e $7^{a}$ Colunas: o desempenho em faixas, em cada uma das três habilidades psicopedagógicas do Teste Ross, ou seja, as habilidades de Análise, Síntese e Avaliação. Pode-se perceber, a partir da análise destas performances nas três habilidades psicopedagógicas, que cada estilo cognitivo de aprendizagem se relaciona com uma tendência de performance nas três habilidades. Por exemplo, no Estilo Analógico-analítico, os participantes que estão inseridos neste agrupamento tem performance Superior (faixa 5) ou Superior ao Termo Médio (faixa 4) na habilidade de Análise. É esta, portanto, a característica fundamental que agrupa estes sujeitos num cluster de similaridade. $\mathrm{O}$ desempenho nas outras duas habilidades psicopedagógicas não se mostra crucial. Já para os integrantes do cluster referente ao estilo Concretogenérico, apresentam performance Inferior (faixa 1) ou Inferior ao Termo Médio (faixa 2) na habilidade de Análise.

Quadro 03: Agrupamentos por Estilo Cognitivo de Aprendizagem

\begin{tabular}{c|c|c|c|c|c|c}
\hline Grupos & \multirow{2}{*}{$\begin{array}{c}\mathbf{N}^{0} \text { de } \\
\text { Sujeitos }\end{array}$} & $\begin{array}{c}\text { \% da } \\
\text { Amostra }\end{array}$ & $\begin{array}{c}\text { \% Cumula- } \\
\text { tiva }\end{array}$ & \multicolumn{3}{|c}{ Habilidades Psicopedagógicas } \\
\cline { 5 - 7 } & & & & $\begin{array}{c}\text { Habilidade 1 } \\
\text { (Análise) }\end{array}$ & $\begin{array}{c}\text { Habilidade 2 } \\
\text { (Síntese) }\end{array}$ & $\begin{array}{c}\text { Habilidade 3 } \\
\text { (Avaliação) }\end{array}$ \\
\hline Analógico- Analítico & 34 & $42,5 \%$ & $42,5 \%$ & 4 ou 5 & - & - \\
Concreto- Genérico & 18 & $22,5 \%$ & $\mathbf{6 5 , 0 \%}$ & 1 ou 2 & - & - \\
Dedutivo- Avaliativo & 14 & $17,5 \%$ & $82,5 \%$ & 3 & 1,2 ou 3 & 4 ou 5 \\
Sintético-Avaliativo & 08 & $10,0 \%$ & $92,5 \%$ & 3 & 4 ou 5 & 4 ou 5 \\
Relacional - Sintético & 02 & $2,5 \%$ & $95,0 \%$ & 3 & 4 ou 5 & 1,2 ou 3 \\
\hline
\end{tabular}

Nota. $N=81$ - No Quadro 03 são apresentados os resultados desta análise, o desempenho e as habilidades psicopedagógicas que são avaliadas da seguinte forma: 5 superior, 4 superior termo médio, 3 termo médio, 2 inferior termo médio, 1 inferior. 


\section{Considerações Gerais Sobre a Nomenclatura dos Estilos Cognitivos de Aprendizagem da Popula- ção da CRT}

A definição da nomenclatura dos cinco Estilos Cognitivos de Aprendizagem gerados a partir da análise de clusters dos dados da aplicação do Teste Ross, bem como da Bateria BPR-5, tem como sua lógica a estipulação do Processo Cognitivo mais característico e relevante do estilo em questão. Este processo cognitivo, por sua vez, influencia o tipo de Habilidade Psicopedagógica predominantemente utilizada pelo indivíduo, quando de seu contato com as demandas cognitivas, de qualquer atividade de aprendizagem com que se defronte.

São os processos cognitivos subjacentes às habilidades Psicopedagógicas, portanto, que alicerçam fundamentalmente os Estilos Cognitivos de Aprendizagem. Com base nos processos cognitivos mais significativos em cada habilidade são construídos os Estilos Cognitivos. Portanto, os Estilos Cognitivos são estabelecidos por meio da avaliação dos processos e das habilidades psicopedagógicas via Teste Ross e confirmados pelos resultados obtidos pelas três provas de raciocínio do BPR-5. Os processos cognitivos e as habilidades psicopedagógicas são determinados pela performance da amostra.

\section{Estilos Cognitivos de Aprendizagem}

Os cinco Estilos Cognitivos de Aprendizagem foram agrupados estatisticamente através da análise de Clusters e tiveram a determinação dos critérios de formação de tais clusters explicados pela interrelação entre o processo cognitivo mais utilizado e que alicerça a habilidade psicopedagógica proeminente para cada um destes Estilos.

A conceitualização, bem como as características de cada Estilo pode ser visualizada a seguir:

Estilo Analógico-Analítico: caracteriza-se por buscar informações utilizando-se de padrões de comparações com o conhecimento prévio; decompor as informações em blocos (análise); possuir alta capacidade de realizar elaborações construindo "pontes" entre conhecimentos novos e os anteriormente adquiridos; possuir alta capacidade e eficiência na compreensão das interrelações; e tende a aprofundar o assunto.
Estilo Concreto-Genérico: o indivíduo do tipo concreto-genérico busca entender os conteúdos de forma linear e sequiencial; trabalha holisticamente com a informação, possibilitando a prática da tarefa; busca exemplificação sistemática para facilitar a memorização e atenção aos procedimentos e detalhes; a memória é uma de suas melhores habilidades intelectuais; trabalha pouco com a abstração de princípios e conceitos; tende a ser pragmático e cuidadoso; e trabalha mais em prol dos objetivos da aprendizagem.

Estilo Dedutivo-Avaliativo: aprende novas informações inferindo um padrão lógico do conteúdo; utiliza-se de uma metodologia na busca de informações; faz análises exaustivas de coerência, validade e veracidade das informações; é sistemático e crítico; tem elevada capacidade de trabalho e atenção; não considera os exemplos concretos; a organização do material é crucial para este tipo de aprendiz.

Estilo Relacional-Sintético: o indivíduo do tipo relacional-sintético reorganiza as subpartes de um conjunto de informações em uma estrutura conceitual integrada e abstrata; revisa com frequiência os conceitos, reformulando-os, relacionando e agregando informações; possui elevada capacidade de abstração e de geração de hipóteses; aprende melhor com abstrações e suas relações; absorve melhor a informação quando são apresentadas com figuras, cores, diagramas, esquemas e demonstrações.

Sintético-Avaliativo: estes indivíduos buscam a aprendizagem de novas informações através de um processo de intercalação entre a visão global dos dados e avaliações específicas dos mesmos dados, de uma forma versátil. Geralmente iniciam analisando a informação como um todo, fazendo uma visão rápida e superficial de todo conjunto informacional. Posteriormente, partem para análises de sub-partes do conteúdo, tentando compreender, pormenorizadamente, cada bloco informacional para fins avaliativos. Possuem boa capacidade de sintetizar os dados, formando um conceito integrador mais amplo.

Tipicamente, são sujeitos com excepcional nível de organização do material de estudo. Tem a preferência por material teórico e são bastante 
sistemáticos na sua forma de exploração do material instrucional. Conseguem com esta estratégia global-específica, um nível bastante profundo de entendimento.

\section{Conclusões}

Geraram-se cinco Estilos. Cognitivos de Aprendizagem: Analógico-analítico, Concretogenérico, Dedutivo-avaliativo, Relacionalsintético e Sintético-avaliativo, através da avaliação dos processos cognitivos e das habilidades psicopedagógicas via Teste de Ross e BPR - 5.

Os resultados obtidos, através das análises de fidedignidade pelo alpha de Cronbach, demonstram que o Teste Ross e os três subtestes da Bateria de Provas de Raciocínio BPR - 5, para a amostra trabalhada, foram altamente discriminativos, consistentes e fidedignos.

Foram apresentadas as adaptações realizadas dos resultados dos testes. Estas foram realizadas com o objetivo de que as especificidades desta população fossem mantidas através da distribuição dos indivíduos em faixa de desempenho e levando-se em conta o desempenho da amostra em diferentes testes, ao invés de se considerar somente o número de questões em cada seção.

Foram identificados os sujeitos característicos de cada um dos Estilos para, futuramente, dentro do cronograma do Projeto "Tapejara", gerar cinco amostras. Pretende-se com isso verificar, posteriormente, as Trajetórias de Navegação que estes indivíduos utilizam ao se defrontarem com tarefas de aprendizagem via ensino computadorizado através da internet.

Por fim, salienta-se que a proposta teóricometodológica do Projeto Tapejara apresenta em seu escopo central um pressuposto bastante moderno, desafiador e revolucionário às concepções mais tradicionais de ensino. Uma das atividades centrais do trabalho de determinação do Modelo de Aluno é justamente conhecer profundamente as Trajetórias de Aprendizagem existentes para cada um dos Estilos Cognitivos de Aprendizagem das populações-alvo do Projeto Tapejara. Para tanto, é necessário a testagem dos usuários de cada Estilo Cognitivo nos Módulos dos Tutores. Evidentemente, que no material instrucional já está acoplado uma Interface padrão, já definida experi- mentalmente na população-alvo, bem como um gravador de Log, que registra as trajetórias dos alunos típicos de cada estilo. Esta interface padrão foi definida a partir de uma pesquisa realizada com os funcionários da empresa de Telecomunicações. Desta forma, foi possível definir uma interface amigável, adequada as características dos alunos e que respeita a ergonomia cognitiva dos usuários (Madeira et al., 2002).

Enfim, a utilização desta metodologia de levantamento dos Estilos Cognitivos (através da investigação dos processos cognitivos) e do estudo das correlações entre Estilos Cognitivos e as trajetórias de aprendizagem possibilitarão, numa etapa subseqüente do Projeto, a modelação cognitiva do aprendiz, gerando-se assim índices (informações) para a modelação do agente-aprendiz (agente computacional) num modo tutorial. A partir da definição de uma forma pela qual o sistema tutorial possa identificar o estilo cognitivo de aprendizagem do aluno, pretende-se a implementação de cursos adaptados aos seus Estilos Cognitivos de Aprendizagem e que realmente possibilitem uma aprendizagem eficaz.

Pelo visto acima surge a necessidade de, em ambientes de ensino (por exemplo, via internet), conhecer-se o Estilo Cognitivo de Aprendizagem dos alunos, a fim de disponibilizar material que realmente se adapte às necessidades deste propósito, sejam elas quais forem, de acordo com os pressupostos da ergonomia cognitiva. Foi justamente neste empreendimento teóricometodológico que os principais resultados obtidos pela pesquisa presentes, neste artigo, se situam e se fundamentam.

Ambientes de ensino que se adaptem ao Estilo Cognitivo de Aprendizagem do aluno levam o mesmo a obter uma aprendizagem mais efetiva e, fundamentalmente, que respeita a individualidade de cada ser humano, o que é sempre fonte de maior eficiência. Obtém-se, com isto, a máxima adaptação dos objetos e eventos (envolvidos no ensino) às características dos indivíduos, saindo-se, portanto, do paradigma taylorista e indo ao encontro do paradigma da Ergonomia Cognitiva, tornando-se, assim, ecológico. É justamente nesta nova concepção do ato de ensinar que o Projeto Tapejara se insere. 


\section{Referências Bibliográficas}

Abrantes, P. (1993). Epistemologia e cognição. Brasília: Editora Universidade de Brasília.

Anderson, J. (1985). Cognitive psychology and its implications. New York: W.H. Freeman.

Bagley, C.; Malick, K. (1998). Field independence, cultural context and academic achievement: a commentary. British Joumal of Educational Psychology.

Bazerman, M.H.; Neale, M.A. (1992). Negotiating rationally. New York: Free Press.

Becker, W.C. (1991). Toward an integration of behavioural and cognitive psychologies through instructional technology. Australian Journal of Educational Technology, 7, (1).

Bloom, B. (1972). Taxionomia dos objetivos educacionais - domínio cognitivo. Porto Alegre: Ed. Globo.

Carnevale, P.J. \& Pruitt, D.G. (1992). Negotiation and Mediation. Annual Review of Psychology, 43, 431-582.

Chanlin, L.J. (1998). Students cognitive styles and the need of visual control animation. Journal of Education Computing Research, 19.

Clarke, M.G. (1998). A study of cognitive styles and fast mapping skill exhibited by middle class black american four year old girl. Dissertation abstracts international; section $B$. The Science and Engineering, 59, (1-B).

Costermans, J. (1981). Psychologie cognitive. Belgique: U.C.L.

Dawes, R.M. (1988). Rational choice in an uncertain world. San Diego: Harcourt Brace Javanovich.

Dunlop, C.E.M. \& Fetzer, J.H. (1993). Glossary of cognitive science. New York: Paragon House.

Gardner, H. (1995). A nova ciência da mente. São Paulo: Edusp.

Genesereth, M.R. \& Nilson, N.J. (1988). Logical foundations of artificial intelligence. Palo Alto: Morgan Kaufmann Publishers.

Ginsberg, M.L. (1993). Essentials of artificial inttelligence. San Mateo: Morgan Kaufmann Publishers.

Hunt, S. K. (1999). Cognition and communication: student's cognitive styles and the argumentation and debate course. Dissertation abstract international. section A Humanities and Social Sciences, $60,(3-\mathrm{A})$.

Kirsh, D. (1991). Foundations of AI: the big issues. Artificial Intelligence, 47, p. 3-30.

Lax, D.A. \& Sebenius, J.K. (1986). The manager as negotiator. New York: Free Press.

Madeira, M.J.P. (1989). Construçāo de modelos de desenvolvimento e da formação de conceitos $\mathrm{cm}$ crianças brasileiras. Projeto de pesquisa em psicologia. Porto Alegre: Pontifícia Universidade Católica do Rio Grande do Sul.

Madeira, M.J.P. \& Filho, J.C.B. (1995). As ciências cognitivas. Veritas, 40 , No. 158 , (p. 233-234).

Madeira, M.J.P. et al. (no prelo). Estilos Cognitivos de Aprendizagem a partir da avaliação de processos mentais superiores e de habilidades psicopedagógicas. San Juan, Porto Rico: Revista da Sociedade Interamericana de Psicologia.

Madeira, M.J.P.; Carneiro J. et al. (2002). Relatório de questões motivacionais. São Leopoldo: Universidade do Vale do Rio dos Sinos, Centro de Ciências da Saúde, Curso de Psicologia.

Madeira et al. (2002). Relatório de resultados: estudos de preferência de interfaces gráficas e tendências de navegação. São Leopoldo: Universidade do Vale do Rio dos Sinos, Centro de Ciências da Saúde, Curso de Psicologia.

Mckay, E. (1999). Exploring the effect of graphical metaphors on the performance of learning computer programming concepts in adult learners: a pilot study. Educational Psychology, 19.

Medin, D.L. \& Ross, B.H. (1992). Cognitive psychology. Orlando: Harcourt Brace Jovanivich College Publishers.

Messick, S. (1976). Individuality in learning. San Francisco: Jossey-Bass.

Minsky, M. (1988). The society of mind. New York: A Touchstone Book.

Nassar, Y.H. (1999). The effects of student's learning and their motivational orientations on their preferences regarding the level of exam according to Bloom's Taxonomy. Dissertation abstract international. section A Humanities and Social Sciences, 60, (1-A).

Oliveira, F.M. (1996). Inteligência artificial distribuída. Anais da N Escola Regional de Informática. Canoas: Gráfica da Universidade Luterana do Brasil, p. 54-71.

Pasquali, L. (1996). Teoria e método de medida em ciência do comportamento. Brasilia: Inep.

Pasquali, L. (1997). Psicometria: teoria e aplicações. Brasília: Ed. Universidade Nacional de Brasília.

Pozo, J. I. (1998). Teorias cognitivas da aprendizagem. Porto Alegre: Artes Médicas.

Primi, R. (2000). BPR - 5: Bateria de Provas de Raciocínio: manual técnico. São Paulo: Casa do Psicólogo.

Rich, E. \& Knight, K. (1994). Inteligência artificial. São Paulo: Makron.

Riding, R. \& Rayner, S. (2000). Cognitive styles and learning strategies - understanding stile differ- 
ences in learning and behavior. London: David Fulton Publishers.

Ross, J.D. \& Ross, C.M. (1997). Teste Ross de processos cognitivos, manual de aplicação. Academic Therapy Publications.

Salvador, C.C.; Alemany, I.G.; Martí, E.; Majós, T. M.; Mestres, M.M.; Goni, J.O.; Gallart, I.S. \& Giménez, E.V. (2000). Psicologia do ensino. Porto Alegre: Artes Médicas.

Schmeck (1988). Perspectives on individual differences, learning strategies and learning styles. New York: Plenum Press, pp.83-100.

Shannon, C.E. (1948). The mathematical theory of communication. The Bell System Technical Journal, 27, p. 379-423.

Sherman, S.J.; Judd, C.M. \& Park, B. (1989). Social Cognition. Annual Review of Psychology, 40, p. 282-326.

Silveira, R.A. (1992). Inteligência artificial em educação: um modelo de sistema tutorial inteligente para microcomputadores. Dissertação de mestrado em educação. Pontifícia Universidade Católica do Rio Grande do Sul, Porto Alegre, RS.
Simon, H.A. (1981). As ciências do artificial. Coimbra: Armênio Amado.

Simon, H.A. \& Kaplan, C.A. (1993). Foundations of cognitive science. Posner, M. I.(Ed.). Foundations of cognitive science. Massachusetts: MIT Press, p. 1-47.

Sternberg. R. (1992). As capacidades intelectuais humanas: uma abordagem em processamento de informaçōes. Porto Alegre: Artes Médicas.

Sternberg. R. (2000). Psicologia cognitiva. Porto Alegre: Artes Médicas.

Stillings, N.A. et al. (1991). Cognitive science: an introduction. Cambridge: MIT Press.

Thompson, L. (1998). The mind and heart of negotiator. New Jersey: Prentice Hall.

Este estudo insere-se no projeto multidisciplinar "Tapejara" - Sistemas Inteligentes de Ensino na Internet elaborado em parceria entre o Curso de Psicologia da UNISINOS com o Instituto de Informática da UFRGS e a empresa de Telecomunicações CRT BrasilTelecom. 
